\title{
Narrow bandwidth wavelength filter by guided-mode resonance
}

\author{
Cedric Lenaerts, Vincent Moreau, Yves Lion, Yvon L. M. Renotte
}

Author Affiliations +

Proceedings Volume 5181, Wave Optics and Photonic Devices for Optical Information Processing II; (2003) https://doi.org/10.1117/12.507490

Event: Optical Science and Technology, SPIE's 48th Annual Meeting, 2003, San Diego, California, United States

Abstract

We study the realization of simple resonant structures allowing to obtain holographic reflection filters under a $45^{\circ}$ incidence for a wavelength of $\lambda=632.8 \mathrm{~nm}$. In particular, we will be interested in the influence of the parameters of the structures on the position and the shape of the resonance peaks. The $45^{\circ}$ incidence takes its origin in the will to include resonant components in substrate mode systems. In the second part of this paper, we use the obtained results to study the realization of an active substrate mode spectral filter by the use of an eletro-optic material: zinc oxide ( $\mathrm{ZnO}$ ). The presented results which obtained by an algorithm based on the Rigorous Coupled Wave Theory. (C) (2003) COPYRIGHT Society of Photo-Optical Instrumentation Engineers (SPIE). Downloading of the abstract is permitted for personal use only.

\section{Citation Download Citation}

Cedric Lenaerts, Vincent Moreau, Yves Lion, and Yvon L. M. Renotte "Narrow bandwidth wavelength filter by guided-mode resonance", Proc. SPIE 5181, Wave Optics and Photonic Devices for Optical Information Processing II, (10 November 2003); https://doi.org/10.1117/12.507490 